\title{
Blazing evanescent grating orders: a spectral approach to beating the Rayleigh limit
}

\author{
C. J. Handmer, ${ }^{1}$ C. Martijn de Sterke, ${ }^{1}$ R. C. McPhedran, ${ }^{1, *}$ L. C. Botten, ${ }^{2}$ M. J. Steel, ${ }^{3}$ and A. Rahmani ${ }^{2}$ \\ ${ }^{1}$ Institute for Photonics and Optical Science (IPOS) and Centre for Ultrahigh-bandwidth Devices for Optical Systems (CUDOS), \\ School of Physics, University of Sydney, Sydney, New South Wales 2006, Australia \\ ${ }^{2}$ Department of Mathematical Sciences and CUDOS, University of Technology, Sydney, New South Wales 2007, Australia \\ ${ }^{3} \mathrm{MQ}$ Photonics Research Centre, CUDOS and Department of Physics and Engineering, Macquarie University, \\ Sydney, New South Wales 2109, Australia \\ *Corresponding author: ross@physics.usyd.edu.au
}

Received April 21, 2010; revised June 7, 2010; accepted June 24, 2010; posted July 20, 2010 (Doc. ID 127346); published August 17, 2010

We develop a way to enhance the amplitudes of the nonpropagating evanescent orders of resonant dielectric gratings. We use this blazing to design gratings with spectra tailored to generate steerable sub-Rayleigh field concentrations on a surface. We investigate the enhancement and customization of evanescent fields necessary to create a virtual and passive scanning probe with no moving parts. Spot size can be decreased 1 order of magnitude below the free-space Rayleigh limit. (C) 2010 Optical Society of America

OCIS codes: $\quad 050.6624,050.1960$.

The wavelength-dependent response of spatially periodic optical structures (gratings) has been exploited for many years, especially in spectroscopy. Gratings can be blazed to favor the reflection or transmission of light into a desired propagating order with high resolving power [1]. The realization that gratings can also excite useful subwavelength evanescent fields, necessary for achieving resolution beyond the Rayleigh limit, has generated a number of proposals [2-4]. Here we develop an approach to enhance ("blaze") and tailor desirable evanescent fields.

The Fraunhofer equation $\sin \theta_{m}=\sin \theta_{0}+m \lambda / d$ governs the angles $\theta_{m}$ at which a grating of period $d$ with an incident plane wave of wavelength $\lambda$ at an angle $\theta_{0}$ generates plane-wave orders. Writing the spatial part of the field as $\exp \left(i k_{x} y+i k_{y} y\right)$, the Fraunhofer equation is cast in terms of parallel $(x)$ and perpendicular $(y)$ components of the incident and scattered wave vectors $(k)$ :

$$
k_{x m}=k_{0} \sin \theta_{0}+2 \pi m / d ; \quad k_{y m}=\left(k_{0}^{2}-k_{x m}^{2}\right)^{1 / 2} .
$$

Here $k_{0}=2 \pi / \lambda$ is the incident wave number and $m$ indices the generated orders. For sufficiently large $|m|$, $\left|k_{x m}\right|>k_{0}$, so $k_{y m}$ is imaginary; the sign is chosen such that these evanescent orders decay exponentially away from the grating. Thus Eq. (1) takes the form $k_{y m} \sim$ $i\left|k_{x m}\right| \approx 2 i \pi|m| / d$, independent of wavelength. This suggests the possibility of sub-Rayleigh resolution, if we can excite these large $|m|$ orders.

By the field continuity conditions, subwavelength field features on the grating surface require orders with subwavelength variation. As these orders are evanescent, this fine detail is not visible in the far field. In the absence of losses or scatterers, the energy in evanescent fields remains trapped on the surface, and so their amplitude is not limited by energy conservation. Thus while blazing propagating orders is well known, blazing evanescent orders should be particularly effective and would provide strong fields at a scale below the Rayleigh limit, an essential feature of superresolution $[\underline{3}, \underline{4}]$. Below, we present a way to blaze evanescent orders and propose a method to exploit this to provide sub-Rayleigh limit resolution.

Our approach requires multiple high- $Q$ internal grating resonances. These resonances, most simply described by guided-mode resonances in a homogeneous dielectric waveguide [5], are due to Fabry-Perot reflection between the grating top and bottom surfaces. Their high $Q$ is due to total internal reflection (TIR), so they couple very weakly to the propagating orders through which energy would otherwise leak away. Rather, they couple strongly to evanescent orders, driving them when on resonance. These resonances are Fano resonances, in which the high- $Q$ resonance is superimposed on a broad background characteristic of a uniform slab.

While Eq. (1) determines the wave vectors of each order, it does not provide the associated amplitudes. To understand blazing we must know these amplitudes and so, settling on a specific geometry, we performed a calculation using the modal method for one-dimensional lamellar gratings [6,7]. Though our approach is general, for convenience we restrict ourselves to $\mathrm{TE}$ polarization. The single dielectric lamellar grating consists of repeating rectangles of two different dielectrics suspended in vacuum, here taken to have refractive indices $n_{\mathrm{L}}=$ $1.46\left(\mathrm{SiO}_{2}\right)$ and $n_{\mathrm{H}}=3.61$ (silicon, $\lambda=1.6 \mu \mathrm{m}$ ); see the inset of Fig. 2(a). While both the phenomenon and the modal method can function with substrates, for the sake of simplicity we focus on the essential physics without a substrate. Because the grating resonances are best defined in the presence of only a single propagating order [8], we chose to use a grating with $d=0.76 \mu \mathrm{m}<\lambda / 2$.

Figure 1 shows the amplitudes of several orders under unit incidence from below versus incident angle. The propagating transmitted (specular) order $(m=0)$ gradually decreases as the angle tends to $90^{\circ}$. This trend is interrupted by Fano resonances, which signify a strong grating resonance, or anomaly, leading to a strong enhancement in the evanescent orders. The different amplitudes of \pm orders, especially near glancing incidence, distinguish this system's asymmetrical enhancements from other diffractive systems, such as a metal sheet with 


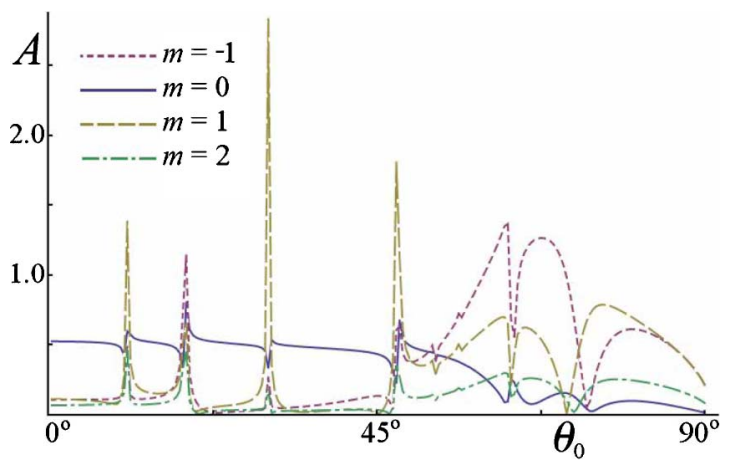

Fig. 1. (Color online) Amplitude of transmitted specular order (solid curve) and three near-evanescent orders versus incident angle, showing evanescent order blazing. Incident angle range $0^{\circ}, 90^{\circ}$ maps to spectral range $0, k_{0}$. Grating is as in the inset of Fig. 2(c).

small holes. This is because the enhancement of each diffracted order is dominated, via TIR, by a particular grating mode resonance.

Even though a single lamellar grating can thus blaze an evanescent order to large amplitudes, this approach has limitations. As these blazed evanescent orders are driven through TIR by resonances of propagating fields in the grating, they are limited to orders for which $\left|k_{x}\right|<n_{\mathrm{H}} k_{0}$. We define such orders as near evanescent. The wavenumber of their field variation is less than $n_{\mathrm{H}} k_{0}$, and they are thus not substantially more useful for sub-Rayleigh resolution than other techniques which use TIR, such as dark field microscopy or immersion microscopy [9].

In contrast, far-evanescent orders $\left(\left|k_{x}\right|>n_{\mathrm{H}} k_{0}\right)$ have no strong connection to the resonances that drive nearevanescent orders. Thus a novel approach is needed to scatter the energy in blazed near-evanescent orders to orders beyond the $\lambda / 2 n_{\mathrm{H}}$ immersion limit. We have discovered that this may be achieved by layering gratings of different periods. Provided the period of the gratings is a simple integer ratio, the structure is periodic and mathematically tractable. In general, the amplitude of evanescent orders is inversely related to $k_{x m}$ and thus limited by the highest amplitude near the middle of the spectrum, typically in a propagating order. This is alleviated by stacking gratings, where a blazed near-evanescent order is the dominant incident field for the short period grating, which then directly scatters efficiently into the desired far-evanescent orders. As the amplitude of nearevanescent orders can be much larger than that of propagating orders, this permits the blazing of farevanescent orders, while skipping intervening, undesired propagating and near-evanescent orders.

This enhancement, the key result in this Letter, is demonstrated in Fig. 2, which contrasts the spectral profile of the single grating [Fig. 2(a)] and the stacked grating [Fig. 2(c)] shown in the insets. Both show the amplitude of the transmitted spectrum on the upper surface of the grating under a circular incident field with unit amplitude per angle [Fig. 2(b)]. Whereas a single grating has practically no energy beyond $n_{\mathrm{H}} k_{0}$, the stacked grating has a spectral envelope stronger than the incident field that extends well into the far-evanescent region. Choices of materials, grating periods, and duty cycle

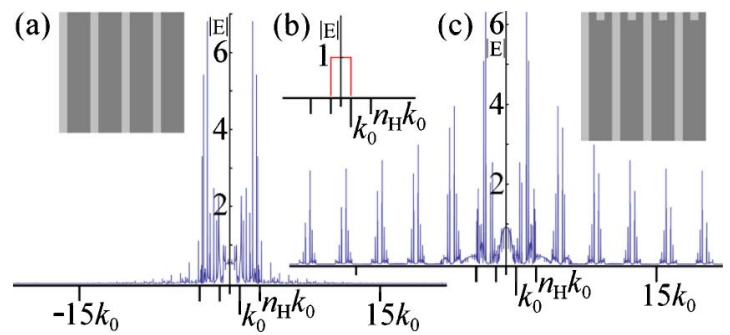

Fig. 2. (Color online) Spectrum on the upper grating surface in transmission of (a) single resonant grating and (c) stacked grating under (b) circular incident field of unit amplitude per angle. The horizontal axis is divided into regions of propagating $\left(0, k_{0}\right)$, near $\left(k_{0}, n_{\mathrm{H}} k_{0}\right)$, and far-evanescent $\left(>n_{\mathrm{H}} k_{0}\right)$ orders. Insets show the geometry of each $\mathrm{Si}$ (dark gray) and $\mathrm{SiO}_{2}$ (light gray) grating; $3.05 \mu \mathrm{m}$ thick, $0.76 \mu \mathrm{m}$ period, $25 \%$ duty cycle. The fine grating in (c) has a thickness of $0.15 \mu \mathrm{m}$.

allow considerable freedom in the frequency and intensity of the far-evanescent peaks.

Strong evanescent fields, efficiently coupled to propagating modes using a stacked grating opens new avenues for high-resolution optical microscopy. For instance, in far field optical diffraction tomography (FFODT), a grating is used to extract high spatial frequency information restricted to the near field of a sample by converting evanescent fields scattered by the sample into propagating fields accessible in the far field [2-4]. FFODT uses an iterative computational approach to reconstruct the entire electric field at the surface from the diffracted far field. While a FFODT prototype was demonstrated [4], its practicality is limited by the strength of evanescent fields that can be outcoupled from the near field of the sample. Hence the resolution of FFODT depends on how much fine control is possible over evanescent fields, particularly far-evanescent orders. Unlike many subdiffraction microscopy techniques [9], FFODT does not require fluorescent dyes, scatterers, or a moving probe.

To illustrate our control over blazed evanescent orders, we create a movable subwavelength optical spot. Its spatial Fourier transform gives the complex spectrum of the orders required to generate this spot; the incident field, interacting with the grating, needs to generate this spectrum. As shown in Fig. 1, only some incident angles lead to blazed evanescent orders; we maximize this number by selecting the grating thickness. These blazed evanescent orders broaden the spectrum of the incident field, which otherwise would consist of propagating plane waves only, leading to a smaller spot. Thus, accessing more remote evanescent orders should enable a further reduction in spot size. Indeed, stacking two gratings with periods of $0.76 \mu \mathrm{m}$ and $0.38 \mu \mathrm{m}$ (period ratio 1:2) broadens the Fourier spectrum (Fig. 2) significantly and decreases the spot size further by a factor of at least 3 . This spot can be used as a source for optical microscopy with a lateral resolution beyond the Rayleigh limit. Subwavelength resolution normal to the grating is ensured by the evanescent nature of the fields.

While such spots are much narrower than the Rayleigh limit, they are not explicitly steerable, as the location of the peaks is fixed by the spectral envelope. However, the spatial envelope can be moved, generating adjacent spots, by adjusting the amplitude and phase of the blazed orders. The Fourier space method provides a heuristic 
(a)

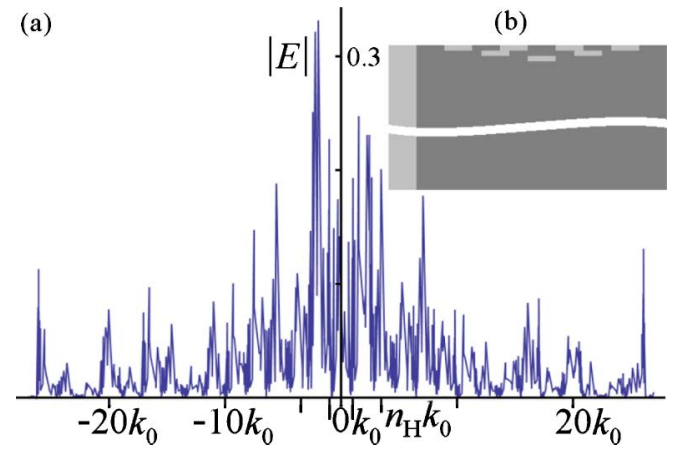

Fig. 3. (Color online) (a) Spectral amplitude of the left peak in Fig. 4. (b) Schematic of upper part of unit cell showing stacked gratings. Primary (coarse) grating dimensions: $3.05 \mu \mathrm{m}$ thick, $0.76 \mu \mathrm{m}$ period, $10 \%$ duty cycle. Fine gratings on the surface have period ratios of $1: 2: 3: 5$, thickness $0.015 \mu \mathrm{m}$ each.

approach to optimize grating design for a scannable narrow spot. We desire closely spaced narrow spots beneath a spatial envelope of comparable width; this demands a stack of subperiod gratings able to create a broad, continuous spectrum from the single grating spectrum shown in Fig. 2(a). A pair of stacked gratings with a period ratio of $1: p(p$ integer) replicates the central paired enhancements seen in Fig. 2(a) outward a distance proportional to $p$. Every $p$ th order is directly connected to the central propagating (specular, $m=0$ ) channel and thus is relatively depleted of energy. All intermediate orders, connected to neither propagating nor near-evanescent orders (modulo $p$ ), are unaffected.

A stack of different subperiod gratings is needed to fill in as much of the spectrum as possible, creating the required spectral profile. The incident field consists of a discrete set of incident plane waves, each exciting a resonance. The complex amplitude of each incident plane wave defines the complex amplitude of the orders it blazes. The transmitted field is chosen such that the sum of the blazed orders forms the closest approximation to the complex spectrum of a narrow spot in its desired location. The parameter space was optimized using a Monte Carlo Markov chain, with a cost function assessing the ratio of energy in the desired versus background regions. Changing the incident field enables scanning of the spot across the grating surface.

Our sample calculation models a stack of silicon/silica gratings with period ratios 1:2:3:5, as in Fig. 3(b). We generate the spots shown in Fig. 4 , which are all well below the Rayleigh limit and span a unit cell of the grating. Figure 3(a) shows the spectral amplitude of the leftmost spot in Fig. 4 ; it has a width of about $3 n_{\mathrm{H}} k_{0}$, a factor $3 n_{\mathrm{H}}$ wider than the free-space envelope. This gives a spot width of $(\lambda / 2) /\left(3 n_{\mathrm{H}}\right) \approx \lambda / 20,1$ order of magnitude smaller than the free-space Rayleigh limit. The robustness of the optimized spots was assessed by randomly modifying

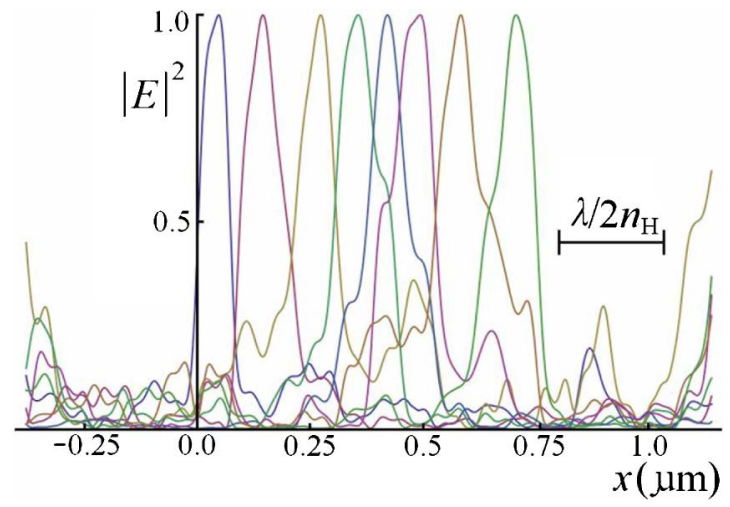

Fig. 4. (Color online) Energy density on the upper grating surface, demonstrating multiple steerable narrow spots independently excitable across a complete period $(0.76 \mu \mathrm{m})$. Each spot is substantially narrower than $\lambda / 2 n_{\mathrm{H}}=0.22 \mu \mathrm{m}$.

the incident field amplitudes by $10 \%$. Although the amplitude of spots varied by up to $30 \%$, the spotlike nature of the fields was preserved. This demonstrates that the spots are due to evanescent-wave interference rather than "superoscillations" [10], which can be sensitive to changes as small as one part in $10^{5}$.

Spectroscopic uses of gratings view grating anomalies such as Fano resonances as nuisances. Here we turn grating resonances to our advantage, demonstrating that stacked dielectric gratings of different periods may be tailored to blaze evanescent grating orders and to produce sub-Rayleigh optical field features in a controllable way.

This work was produced with the assistance of the Australian Research Council. The authors thank Hugues Giovannini and Patrick Chaumet for useful discussions.

\section{References}

1. R. Petit, Electromagnetic Theory of Gratings (Springer, 1980).

2. P. C. Chaumet, K. Belkebir, and A. Sentenac, Opt. Lett. 29, 2740 (2004).

3. A. Sentenac, P. Chaumet, and K. Belkebir, Phys. Rev. Lett. 97, 243901 (2006).

4. G. Maire, F. Drsek, J. Girard, H. Giovannini, A. Talneau, D. Konan, K. Belkebir, P. C. Chaumet, and A. Sentenac, Phys. Rev. Lett. 102, 213905 (2009).

5. S. Fan and J. D. Joannopoulos, Phys. Rev. B. 65, 235112 (2002).

6. L. Botten, R. McPhedran, J. Adams, J. Adrewartha, and M. Craig, Opt. Acta 28, 413 (1981).

7. L. Li, J. Opt. Soc. Am. A 10, 2581 (1993).

8. E. Popov, L. Mashev, and D. Maystre, J. Mod. Optics 33, 607 (1986).

9. Nat. Photonics 3, 361 (2009) (editorial), and references therein.

10. F. M. Huang, Y. Chen, F. J. G. de Abajo, and N. I. Zheludev, J. Opt. A 9, S285 (2007). 